\title{
Análisis de los supuestos sobre la migración internacional en las proyecciones de población de México 2001-2050 y 2006-2050
}

\author{
Víctor Manuel García Guerrero*
}

Distintos ejercicios prospectivos consideran que la proyección de la migración está relacionada con la presunción del éxito en diversos objetivos coyunturales de carácter económico, de ahi que en este trabajo se analicen los supuestos de proyección referentes a esta componente demográfica vinculándolos con el marco teórico subyacente y con el contexto político-económico. Se analizan las fuentes citadas en el documento metodológico y en la literatura relacionada con el tema. Algunos de los hallazgos indican que los ejercicios prospectivos examinados suponen un crecimiento económico sostenido de $5 \%$ durante la primera década del siglo XXI, con lo que la migración neta crecería a una tasa constante anual cercana a 0.22. Este supuesto está directamente relacionado con las expectativas de las coyunturas político-económicas de los dos primeros gobiernos mexicanos del siglo XXI y con el crecimiento económico alcanzado en el último quinquenio del siglo pasado. Para incrementar la confiabilidad en sus resultados se propone el uso de técnicas estocásticas para medir adecuadamente la incertidumbre asociada a la migración e incrementar así la objetividad en su proyección.

Palabras clave: proyección de la migración internacional, supuestos de proyección, migración internacional, proyecciones de población, economía y demografía.

Fecha de recepción: 12 de marzo de 2012.

Fecha de aceptación: 10 de junio de 2013.

\section{Analysis of the Assumptions of International Migration in Mexican} Population Projections 2001-2050 and 2006-2050

Several prospective exercises consider that the projection of migration is linked to the presumption of success in various specific economic goals. This paper therefore analyzes the projection assumptions referring to this demographic component by linking them to the theoretical framework and the political-economic context. It analyzes the sources quoted in the methodological document and the literature related. Some of the findings indicate that the prospective exercises examined assume sustained growth of $5 \%$ during the first decade of the 21st century, whereby net migration would grow at a constant

* Profesor investigador del Centro de Estudios Demográficos, Urbanos y Ambientales de El Colegio de México. Correo electrónico: <vmgarcia@colmex.mx>, <vicmgg@ gmail.com>.

$\overline{\text { ESTUDIOS DEMOGRÁFICOS Y URBANOS, VOL. 29, NÚM. } 2 \text { (86), 2014, 229-256 }}$ 
annual rate of nearly 0.22. This assumption is directly linked to the expectations of the political and economic situations of the first two Mexican governments in the 21st century and to the economic growth achieved during the last five years of the 20th century. In order to increase the reliability of its results, the use of stochastic techniques is proposed to accurately measure the uncertainty associated with migration and thereby increase the objectivity of its projection.

Key words: projection of international migration, projection assumptions, international migration, population projections, economics and demography.

\section{Introducción}

Una proyección de población es el cálculo de un escenario hipotético de lo que ocurriría en la demografía de un país o región si determinadas circunstancias políticas, económicas, demográficas y sociales ocurriesen. Tales proyecciones figuran entre los instrumentos más importantes de la planificación gubernamental, ya que son una referencia para los gobiernos sobre las demandas futuras de su población, que están directamente relacionadas tanto con su volumen, con su distribución por edades y con su sexo; por ejemplo, los niños tienen necesidad de educación y acceso a los servicios de salud, los jóvenes y adultos requieren trabajo y seguridad social, y para los adultos mayores es indispensable acceder a los servicios de salud y disponer de una pensión que les garantice su sobrevivencia.

Desde la década de los sesenta se ha realizado aproximadamente una decena de proyecciones de población para México (García Guerrero, 2014, pp. 124-198). En el año de 1983 el Conapo, la Secretaría de Programación y Presupuesto (SPP) y el Centro Latinoamericano de Demografía (Celade) realizaron un trabajo conjunto para proyectar la población mexicana para el periodo 1980-2000 (SPP, Conapo y Celade, 1983). En estas proyecciones se introdujo el concepto de "proyección programática”, el cual, según Partida y Tuirán (2002: 27) se refiere a un tipo de proyecciones demográficas en las que se incorporan las metas de fecundidad establecidas en el Programa Nacional de Población (PNP) de un sexenio determinado "sin que ello implique que el gobierno mexicano haya adoptado como meta de mediano y largo plazos la trayectoria [...] prevista" para los años subsecuentes. Es decir, para estos autores las metas programáticas son sexenales, de corto plazo y exclusivamente relacionadas con la fecundidad. 
Sin embargo, las acciones que se emprenden para modificar los niveles y tendencias de la dinámica demográfica en un momento determinado tienen un impacto en el corto, mediano y largo plazos. La demografía tiene la propiedad de responder más lentamente a los factores que la determinan respecto a algunos otros fenómenos, como los económicos, a menos de que esos factores de cambio sean de alto impacto, como una guerra o epidemia. Por ello, cuando se establecen y ejecutan políticas para cambiar la dinámica demográfica en cierto momento, algunos de estos cambios se producirán en el corto plazo, pero otros ocurrirán en el mediano o largo plazos.

El método para proyectar la demografía es conocido como el de las componentes demográficas. Esta metodología ha sido utilizada en todo el mundo por muchas agencias, como la División de Población de Naciones Unidas y el Conapo. En términos generales, el método de las componentes consiste en proyectar por separado la dinámica de la mortalidad, la de la fecundidad y la de la migración interna e internacional, de tal forma que sus resultados sirvan de insumo para proyectar las cohortes futuras de la población total por sexo y edades.

En virtud de la importancia que tiene el fenómeno migratorio para la demografía mexicana, desde la década de 1990 el Conapo ha aplicado la versión multirregional del método de las componentes, desarrollada por Andrei Rogers (1995). Esta metodología permite simular la interacción entre las personas nacidas en México que viven en el país, los mexicanos residentes en Estados Unidos y los extranjeros residentes en México (Conapo, 2003: 63).

En el método de las componentes subyace un enfoque determinista, ya que no se cuantifica la incertidumbre inherente a cualquier variable que se quiera pronosticar. Por ello no es posible incorporar intervalos de confianza o rangos de variabilidad que tengan asociada una medida de probabilidad. Ante esta deficiencia, se han incorporado en los ejercicios de proyección simulaciones de variantes alternativas a la proyección media. Para su cálculo se suponen trayectorias predefinidas para la fecundidad, la mortalidad y la migración. Por ejemplo, al suponer una fecundidad alta combinada con una mortalidad baja y un saldo neto migratorio "bajo" (cercano a cero) se determina un escenario de alto crecimiento poblacional; con ello se establece la variante alta o cota superior de la proyección media. De igual manera, si se supone una fecundidad baja combinada con una mortalidad alta o con pocos incrementos y un saldo neto migratorio "alto" 
y negativo, se obtiene un escenario de bajo crecimiento poblacional o la variante baja. Sin embargo se ha demostrado que esta estrategia metodológica tiene varios problemas: 1 ) por un lado implica suponer que existe una correlación perfecta entre las variables demográficas a lo largo del tiempo; 2) no es posible determinar la probabilidad de que un evento ocurra dentro de las cotas que se determinan, y 3) son intrínsecamente inconsistentes en el sentido de que representan de forma inadecuada la incertidumbre relativa de distintos indicadores, como el volumen poblacional, la tasa global de fecundidad, las razones de dependencia, etcétera (Lee, 1998: 156).

En su versión determinista, el método de proyección permite incorporar supuestos o metas para alguna o todas las componentes demográficas. Por ejemplo, en las proyecciones del periodo 2006-2050 se supuso que la fecundidad nacional se estabilizaría a partir del año 2035 en aproximadamente 1.85 hijos por mujer, lo cual es muy controvertido. No se ha desarrollado teoría alguna que respalde el establecimiento de valores asintóticos para las variables demográficas y más bien se trata de una estrategia metodológica para evitar que la tasa global de fecundidad (TGF) se acerque mucho a cero.

Los supuestos que se han utilizado desde el inicio de la historia de las proyecciones de población, así como su certeza, dependen completamente de la experiencia, de los conocimientos y, hasta cierto punto, de la sensibilidad del demógrafo. Esta flexibilidad del método se debe a que en sus orígenes no había computadoras que permitieran el cálculo intensivo de miles de escenarios probables; sin embargo, hoy en día sería posible prescindir de dicha subjetividad o se podría capitalizar por medio de técnicas estadísticas más poderosas, como las bayesianas.

En los documentos metodológicos sobre las proyecciones de población de los años 2002 y 2008 (Conapo, 2003 y 2008) no quedan claros los argumentos sobre los supuestos de la migración internacional futura ni los de sus variantes, lo cual es necesario para evaluar si, al menos para esa componente, la metodología proyectiva ha sido utilizada para calcular el escenario demográfico más factible en función de las metas coyunturales que se persiguen.

En virtud de lo anterior, el presente artículo tiene como objetivo analizar los supuestos utilizados para proyectar la migración internacional de México y, a partir de ello, determinar su factibilidad en la proyección del total poblacional. Con ese propósito se analizan los documentos metodológicos de las proyecciones nacionales de población para los periodos 2001-2050 y 2006-2050 y sus referencias. 
Así, el artículo se encuentra estructurado de la siguiente forma: en primer lugar se analizan los supuestos sobre la migración en los ejercicios prospectivos de 2002 y de 2006; se analizan, en el contexto de los periodos sexenales que les corresponden, sus motivaciones e implicaciones y se señalan los marcos teóricos que subyacen; después se analizan las variantes de proyección en torno a la migración internacional, y finalmente se concluye este trabajo con algunas reflexiones finales y propuestas metodológicas alternativas.

\section{Los supuestos sobre la migración en las últimas proyecciones demográficas}

En el Estudio Binacional México-Estados Unidos se especifica que en el año 1996 hubo entre 7 y 7.3 millones de residentes en Estados Unidos de origen mexicano, de los cuales entre 2.3 y 2.4 millones no tenían documentos de residencia (U.S. Commission on the Immigration Reform, 1997). Durante la década de los noventa la cantidad de migrantes de origen mexicano tuvo un incremento promedio anual de entre 277 mil y 340 mil (Escobar et al., 1999: 9). Éste es un cambio importante respecto al incremento promedio anual de esta población en los años ochenta, el cual osciló entre los 210 mil y 260 mil mexicanos (Alba, 2006: 280). Hacia el segundo quinquenio del siglo XXI se estimó que vivían en Estados Unidos alrededor de 12 millones de mexicanos (datos del Pew Hispanic Center citado por Durand, 2006: 13), de los cuales poco más de cinco millones se encontraban en una situación considerada como "irregular" (CMMI, 2005: 35). En este contexto el Conapo estimó que entre los años 2000 y 2005 el incremento promedio anual pasó de 572 mil a 581 mil mexicanos (Conapo, 2006), más del doble del que hubo durante la década de los ochenta. Hacia el final del primer decenio del siglo XXI se estima que la migración neta disminuyó a cerca de 150 mil salidas anuales del país (Passel, 2011: 18).

En el documento metodológico de las proyecciones de población de México se expone que

Las previsiones del comportamiento de la migración internacional son más difíciles de prefigurar debido a las condiciones que rigen a este fenómeno; por ello preferimos suponer que los niveles recientes de la movilidad territorial externa del país, que se encuentran históricamente entre los más elevados, se mantendrán constantes a lo largo del horizonte de la proyección, en lugar de establecer hipótesis cuestionables sobre la evolución 
futura de los complejos y variados factores que inciden en la migración externa de México, sobre todo del cuantioso éxodo de mexicanos hacia Estados Unidos [Conapo, 2008: 122, cursivas propias]. ${ }^{1}$

Sin embargo, más adelante en el mismo documento se apunta que son las tasas de emigración las que se mantienen constantes entre los años 2005 y 2010 (Conapo, 2008: 125). Ese fraseo no aclara si son los niveles o las tasas las que permanecerán constantes. Al graficar los datos tanto de las tasas como de los volúmenes (gráficas 1 y 2), puede pensarse que fueron los niveles de la emigración mexicana los que se mantuvieron constantes; sin embargo a lo largo del documento metodológico se asegura que fueron las tasas de emigración. El pequeño aumento en el saldo neto migratorio entre 2005 y 2010 que se observa en la gráfica 2 puede deberse a la proyección de la inmigración, pero no es posible confirmar esta aseveración debido a que el Conapo sólo publicó la proyección de la migración neta internacional. Desafortunadamente ésta es una mala práctica que debería subsanarse en posteriores ejercicios prospectivos.

El argumento principal para mantener las tasas de emigración constantes a lo largo del periodo de proyección hace referencia a los resultados obtenidos en los trabajos de Tuirán, Partida y Ávila (2000a y 2000b).

\section{Crecimiento económico y migración}

Con el fin de explorar algunos de los efectos posibles de mediano y largo plazos sobre la migración internacional, Tuirán, Partida y Ávila (2000a y 2000b) construyeron algunos escenarios que descansan en el supuesto de que cuando las economías están integradas, los ciclos de expansión y contracción tienden a correlacionarse negativamente. En otras palabras, el supuesto general que subyace en el modelo de estos autores es que los periodos de contracción económica en el país de origen coinciden con los ciclos de expansión económica del país de destino, y viceversa. Con esta articulación se maximizan los incentivos a la migración y se producen los más altos niveles de emigración (Tuirán et al., 2000b: 58). La construcción de cada escenario considera la dirección en la que operan las variables independientes y simula sus

\footnotetext{
${ }^{1}$ Este mismo argumento fue utilizado para la proyección de la componente migratoria del periodo 2000-2050 (Conapo, 2003: 74).
} 


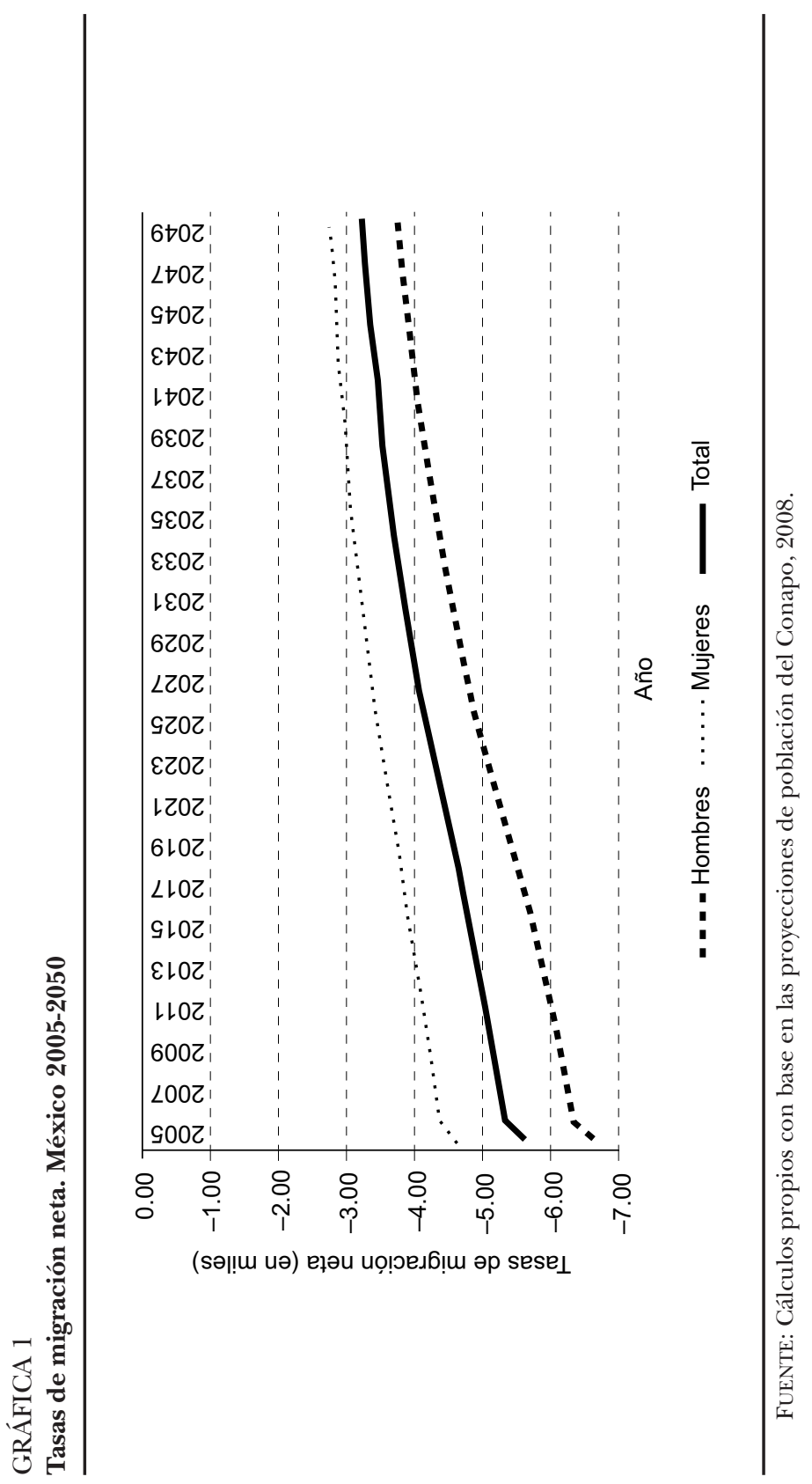




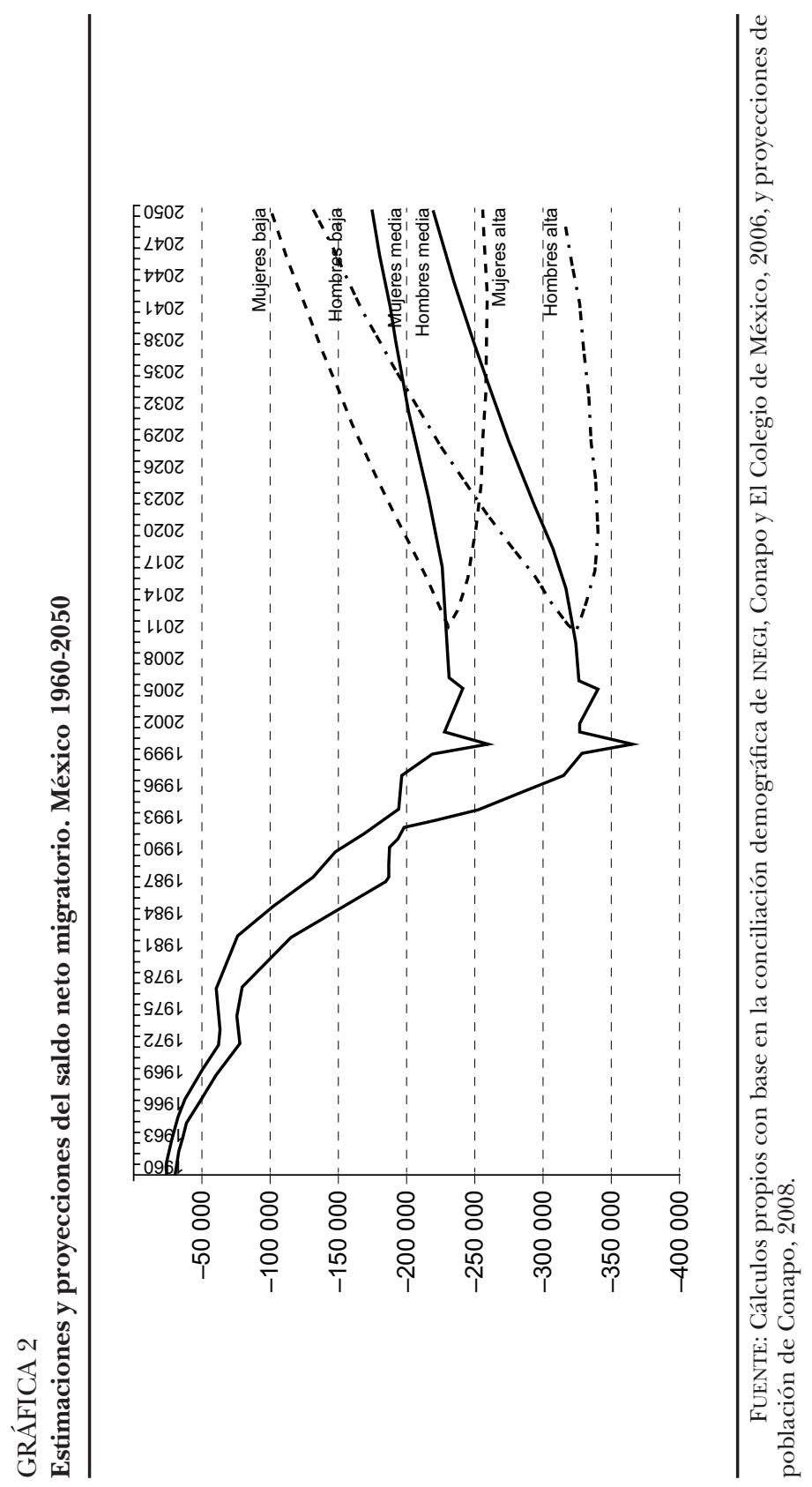


posibles valores durante un horizonte de 34 años (de 1997 a 2030). El modelo planteado por los autores está basado en la teoría económica neoclásica de la migración y, en cierta medida, en la teoría de las redes sociales.

La teoría más vieja y mejor conocida para explicar la migración internacional es la teoría económica neoclásica, la cual tiene dos enfoques: el macro y el micro. En el enfoque macro el análisis se centra en las economías nacionales, regionales o globales; en cambio, en el micro se orienta a los individuos. Se utiliza principalmente para explicar el origen de las migraciones internacionales. En su versión macro, la teoría neoclásica fue desarrollada originalmente para explicar la migración laboral en el proceso de desarrollo económico. Esta teoría establece que la migración internacional es causada por diferencias geográficas en la oferta y la demanda de trabajo. Se dice que los países con una gran dotación de trabajo relacionado con el capital se caracterizan por un bajo mercado salarial; en cambio los países donde escasea la dotación de fuerza laboral relacionada con el capital se caracterizan por un alto mercado salarial (Massey et al., 2000: 7-9).

Así, la teoría neoclásica ofrece una explicación sencilla sobre el inicio del proceso de migración internacional. Esta perspectiva contiene varias preposiciones, entre las que destacan: 1) la migración de trabajadores es causada por la diferencia de salarios entre países; 2) la eliminación de dichas diferencias salariales termina con los flujos laborales; 3) los gobiernos pueden controlar los flujos migratorios por medio de los mercados de trabajo tanto de los países expulsores como de los receptores (Massey et al., 2000: 7-9).

Conforme a la versión micro, la teoría neoclásica ve al individuo como un actor racional que decide migrar o no en función del resultado de un cálculo de costo-beneficio, principalmente monetario. Entre los principales postulados de esta versión destacan los siguientes: 1) los movimientos internacionales provienen de diferenciales internacionales de ingreso y de tasas de desempleo; 2) las características individuales, las condiciones sociales o las tecnologías que bajan los costos de la migración incrementan los rendimientos netos de ésta, y por ello incrementan la probabilidad de la migración; 3) los flujos agregados de migración entre países son la suma de los movimientos individuales; 4) el movimiento internacional no ocurre en ausencia de diferencias de ingresos o tasas de empleo entre países, es decir, la migración ocurre en tanto que los ingresos esperados se hayan igualado internacionalmente, y el movimiento no cesa hasta que este pro- 
ducto se equipare, y 5) los gobiernos controlan la inmigración primeramente mediante políticas que afectan los ingresos esperados en los países expulsores o receptores (Massey et al., 2000: 9-11).

De esta manera, cuando la migración es analizada de acuerdo con la teoría neoclásica, básicamente se supone que se origina por un diferencial salarial macroeconómico que lleva a los individuos a analizar los costos y beneficios de migrar; si el resultado de dicho balance es positivo, entonces se decide hacerlo. De igual manera, se supone que los gobiernos influyen o controlan la migración de manera indirecta, influenciando o controlando algunas variables económicas.

Por otra parte, la teoría de las redes sociales es uno de los marcos teóricos más utilizados para explicar la "perpetuación" o momentum de la migración internacional. Las redes sociales se definen como "conjuntos de lazos interpersonales que conectan a los migrantes, primeros migrantes y no-migrantes en las áreas de origen y destino mediante lazos de parentesco, amistad y de un origen común compartido. Incrementan la probabilidad del traslado internacional porque bajan los costos y los riesgos del movimiento e incrementan los rendimientos netos esperados de la migración" (Massey et al., 2000: 26-27). Entre los principales postulados de esta teoría destaca que: $a$ ) una vez iniciada la migración, ésta tiende a expandirse en el tiempo hasta que las conexiones de la red han alcanzado un nivel máximo, de tal forma que hace posible que todos los individuos del lugar de origen puedan migrar; entonces la migración comienza a desacelerarse; $b$ ) el volumen del flujo migratorio entre dos países no está tan fuertemente relacionado con los diferenciales salariales o tasas de empleo, y $c$ ) el proceso de formación de redes está fuera del control de los gobiernos y ocurre sin importar el tipo de políticas que se apliquen.

Según Tuirán, Partida y Ávila (2000b: 55) "uno de los acontecimientos más relevantes, con importantes implicaciones para la evolución futura de la migración, ha sido la entrada en vigor del Tratado de Libre Comercio (TLC)". Los autores observan que un supuesto implícito en las negociaciones del TLC fue que la promoción del libre movimiento de bienes y capitales contribuiría a sustituir la movilidad de la fuerza de trabajo. Este argumento también se encuentra en el trabajo de Alba (2004), quien asegura que la aceptación política del TLC descansó principalmente en un convencimiento de que afectaría de manera colateral la emigración de mexicanos hacia Estados Unidos. Lo anterior suscitó una discusión entre algunos analistas mexicanos, quienes argumentaban que si las políticas comerciales se dirigían hacia 
la apertura y la liberalización, las políticas migratorias deberían orientarse en el mismo sentido (Jorge G. Castañeda citado por Alba, 2004: 215). Es decir, se cuestionaba que se esperara que el modelo económico resolviera la problemática de manera colateral, pues ésta era una política incompleta, por lo que se tendrían que establecer políticas específicas para la migración.

De esta manera, Tuirán, Partida y Ávila (2000b) establecen cuatro hipótesis que sugieren que dichas reformas: 1) pueden guiar a una reducción del flujo de trabajadores, 2) pueden contribuir a una aceleración de dicho flujo, 3) pueden no tener efecto alguno sobre la migración, o 4) pueden tener efectos diferenciales en el tiempo sobre el flujo de trabajadores migrantes. Respecto a este último punto, los autores plantean que en el corto y mediano plazos las reformas económicas podrían contribuir a incrementar la migración desde México debido a que pueden dar lugar a un desplazamiento de la fuerza de trabajo de los sectores más vulnerables; aunque esta migración tendería a disminuir conforme el libre comercio contribuyese a reducir las disparidades económicas y el diferencial salarial entre ambos países. $\mathrm{Al}$ respecto Phillip Martin observa que

la continuidad de la migración, incluso su crecimiento en el corto y mediano plazos, no son, sin embargo, incompatibles con los resultados migratorios esperados -su disminución- en el largo plazo. Este comportamiento transitorio de aumento a medida que se produce un desarrollo de consideración ha sido descrito como la "joroba migratoria" ya que en un primer momento las corrientes migratorias se incrementan por encima de su trayectoria secular (ante una integración económica más estrecha que implica procesos de ajuste económicos significativos, a la vez que disponibilidad de ingresos corrientes que permiten costear la migración) para después, en el mediano y largo plazos, en un segundo momento, disminuir una vez transpuesto un cierto umbral de desarrollo económico [Phillip Martin, 1993 y 2001, citado por Alba, 2004: 225].

Este argumento muestra que la cuarta hipótesis de Tuirán, Partida y Ávila se enmarca en el modelo de la "joroba migratoria". De acuerdo con Alba (2004), la transición hacia el nuevo modelo de desarrollo ha implicado reacomodos y ajustes, así como un deterioro en las condiciones de vida de los habitantes. Por ello, ante los grandes rezagos que se acumularon en el pasado, la solución al problema del desempleo será de largo plazo y dependerá de una reactivación del proceso de crecimiento económico que conlleve a un incremento gradual del 
empleo, la productividad y los salarios. Sin embargo observa también que México se encuentra en el umbral de una nueva geografía de la producción, la cual traerá una nueva geografía de las migraciones. El camino que transite el desarrollo regional seguirá siendo muy importante en la configuración de dicha geografía. De acuerdo con la forma que adopte el camino del desarrollo se tendrá mayor certidumbre sobre los efectos de mediano y largo plazos de los patrones migratorios. En este marco se advierte que de acuerdo con las previsiones económicas México podría haber experimentado un crecimiento cercano a $5 \%$ en los primeros años del siglo XXI. Esta hipótesis coincide con las metas de crecimiento que fueron trazadas al inicio del gobierno de Vicente Fox. Con dicho ritmo de crecimiento la economía debería haber sido capaz de generar empleos suficientes para el gran número de trabajadores que se sumaron a la fuerza laboral cada año y para reducir el problema del subempleo.

En este contexto, el modelo de Tuirán, Partida y Ávila (2000a y 2000b) -modelo TPA- supone dos condiciones: las que operan en el sentido de aliviar las presiones migratorias y las que las agudizan. Los valores simulados se incorporan a una ecuación de regresión lineal que tiene como variable dependiente la suma de las tasas de emigración por edad estimadas para cada año calendario durante el periodo de 1997 a 2030, y las variables independientes son:

1. La tasa de crecimiento anual del producto interno bruto (PIB).

2. La razón del salario promedio por hora en el sector no agrícola de Estados Unidos entre el pago por hora en el sector manufacturero en México (WAGERATIO).

3. La razón de desempleo abierto de Estados Unidos y México (UNEMPLR).

4. El logaritmo natural del monto anual de las remesas monetarias per cápita (LREMPERC). ${ }^{2}$

5. La interacción entre el logaritmo de las remesas per cápita y la tasa de desempleo abierto en México (LREM-UNEMPLR).

6. La interacción entre el PIB y la tasa de desempleo abierto en México (PIB-UNEMPLR).

Con la primera variable independiente se intenta captar el crecimiento de la economía, con la segunda y tercera se pretende apreciar

${ }^{2}$ El monto de las remesas per cápita se calculó en función de la población que nació en México y reside en Estados Unidos. 
las condiciones económicas de México vis a vis con las de Estados Unidos, con la cuarta se trata de percibir la acción de las redes sociales ${ }^{3}$ y la intensidad de los vínculos entre los lugares de origen y de destino, y acerca de las dos últimas tan sólo se menciona que son relevantes desde los puntos de vista estadístico y sustantivo (Tuirán et al., 2000a: 31-33). Así, y en virtud de lo anteriormente expuesto, el modelo propuesto por los autores se encuentra enmarcado dentro de un enfoque predominantemente neoclásico; con las tres primeras variables se está incorporando la perspectiva neoclásica de la migración y con la cuarta se intenta añadir la perspectiva de las redes sociales.

Por el lado metodológico, no se indica si se realizaron pruebas de multicolinealidad, autocorrelación, permanencia estructural, etc., que formalmente se calculan con cualquier regresión lineal; además, no se ofrece explicación alguna sobre las elasticidades relacionadas, en especial las de las variables de interacción. El único indicador que calculan es el coeficiente de determinación, el cual indica que obtienen una buena estimación de la emigración acumulada $\left(R^{2}=0.82\right)$. En el cuadro 1 se muestran los parámetros estimados con el método de mínimos cuadrados ordinarios.

Así, se observa que el signo asociado a la variable de desempleo no opera en la dirección esperada; es decir, por cada unidad que se incrementa la tasa de desempleo la emigración disminuye. Es por ello que cuando los autores introducen variables colineales (las variables de interacción), la vuelven no significativa y, de esta manera, se encuentra el sentido deseado. Estrictamente, tampoco debería incluirse la variable PIB si se considera un nivel de significancia de 0.05 ya que debe estar explicada en cierta medida por la variable PIB-UNEMPM. Sin embargo, no fue eliminada del modelo porque dejaría de hacer directamente observable el efecto del crecimiento económico en la emigración acumulada.

Ahora bien, respecto a las simulaciones de escenarios alternativos de desarrollo se supuso que: 1) el nuevo modelo económico sería estimulado por el TLC y podría retomar la senda de crecimiento alto y sostenido, como el observado durante 1954-1970; a este escenario se le denominó de "economía alta"; 2) en el segundo escenario, denomi-

${ }^{3} \mathrm{El}$ analizar el efecto de las redes sociales en la migración por medio de las remesas puede producir una explicación un tanto errada. En el trabajo de Tuirán, Santibáñez y Corona (2006) se afirma que el flujo de remesas no es destinado en su totalidad hacia el consumo familiar o hacia la comunidad de origen, sino que una parte muy importante está compuesta por dinero de dudosa procedencia o se utiliza para el pago de "coyotes" y "polleros". 
CUADRO 1

Coeficientes de regresión para la suma de las tasas específicas de emigración, 1997-2030

\begin{tabular}{|c|c|c|}
\hline Variables & Coeficientes $B$ & Valores $T$ \\
\hline PIB & $-0.0031 * *$ & -1.855 \\
\hline WAGERATIO & $0.0050 *$ & 3.958 \\
\hline UNEMPLR & -0.0045 & -0.872 \\
\hline LREMPERC & $0.0198^{*}$ & 5.046 \\
\hline LREM-UNEMPM & $-0.0009 *$ & -2.794 \\
\hline PIB-UNEMPM & $0.0006^{* *}$ & 2.08 \\
\hline Constante & $0.1152^{*}$ & 4.306 \\
\hline $\mathrm{R}^{2}$ & 0.8255 & \\
\hline $\mathrm{R}^{2}$ ajustada & 0.7731 & \\
\hline Observaciones & 27 & \\
\hline $\begin{array}{rl}* & p \leq 0.01 \\
* * & \leq 0.05 \\
* * * & \leq 0.1\end{array}$ & & \\
\hline
\end{tabular}

nado "economía baja", se supuso un desempeño económico errático como el observado desde 1985. Cada escenario fue combinado con dos hipótesis sobre el comportamiento futuro de las remesas: $a$ ) el monto de las remesas se mantiene constante a partir de 1996; $b$ ) el monto de las remesas se incrementa de acuerdo con una función logística. ${ }^{4} \mathrm{Un}$ último escenario, utilizado como referencia, es calculado manteniendo constantes las tasas de emigración estimadas para 1996.

De lo anterior, encuentran cuatro escenarios de cambio futuro de la migración, cuya dinámica se observa en la gráfica 3.

El escenario "A" se refiere a una economía alta y remesas constantes, el "B" a una economía baja y remesas constantes, el "C" a una economía alta y remesas que aumentan, y el "D" a una economía baja y remesas que aumentan. De esta manera, los autores concluyen que el escenario de tasas constantes genera resultados intermedios a los

${ }^{4}$ El valor de la asíntota inferior es de 585.9 dólares de Estados Unidos y el de la superior de 969.81 dólares; la primera corresponde al nivel observado en 1996, pero no queda claro cómo se determina el valor para la asíntota superior. 


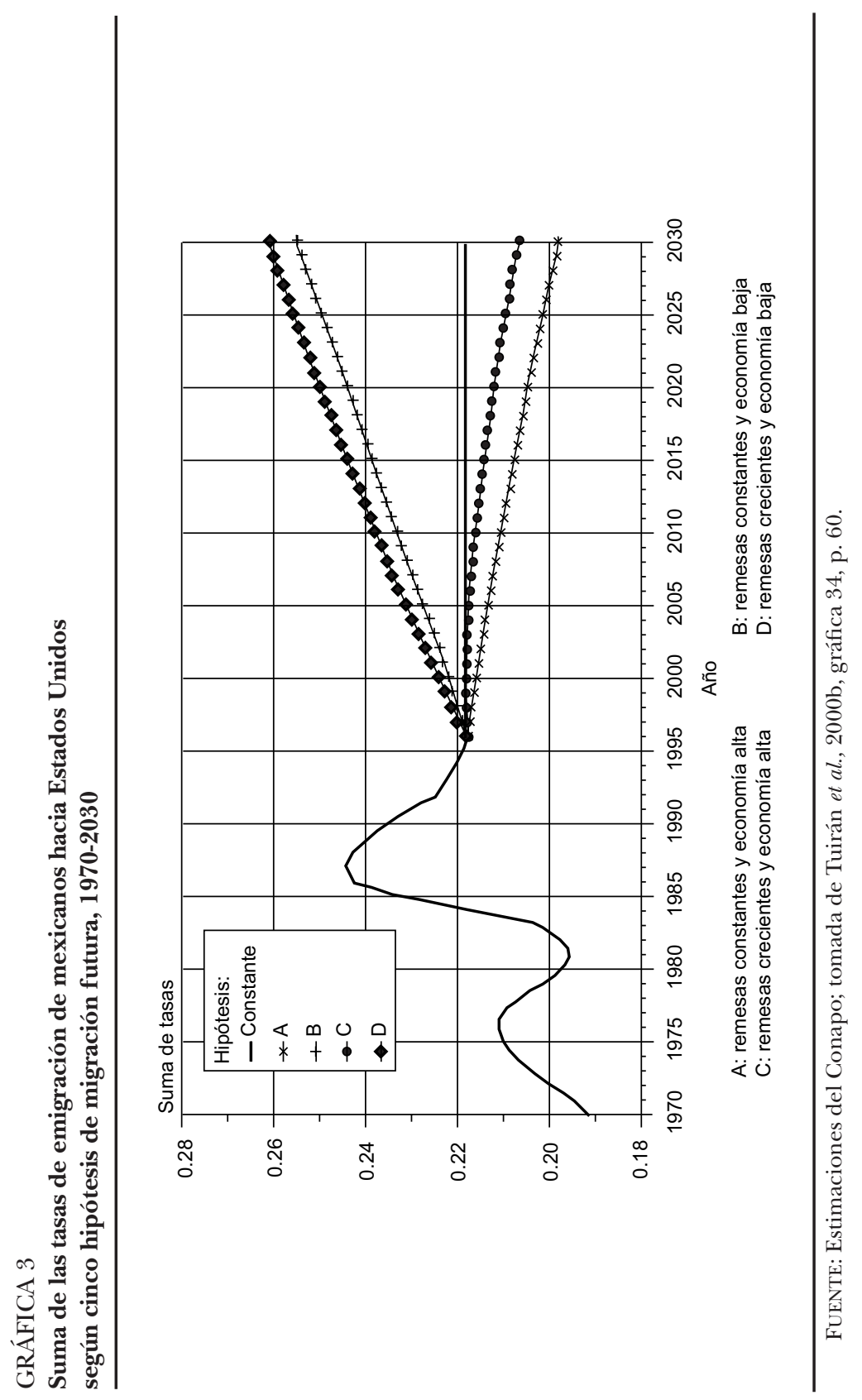


que arrojan los escenarios alternativos; sin embargo, éste se encuentra más cercano a los obtenidos en los escenarios "A" y "C", los cuales se refieren a dos escenarios optimistas respecto al crecimiento económico. Es decir, el escenario de tasas constantes se encuentra más próximo a las metas de desarrollo planteadas por el gobierno mexicano en 2006. En la gráfica 2 se muestra que en el corto plazo los niveles de migración son casi iguales a los que se observarían en los escenarios más optimistas de crecimiento económico, y en el mediano y largo plazos la brecha tiende a incrementarse. Este escenario es de especial importancia debido a que es el que fue incorporado en las proyecciones de población. En virtud de lo anterior es posible afirmar que el supuesto que realmente se encuentra detrás de la proyección de la migración internacional es que el crecimiento económico estaría cerca de $5 \%$ en el corto plazo.

Como se mencionó, en las proyecciones poblacionales para 20012050 se supuso que la tasa de emigración sería constante a lo largo del horizonte proyectivo. Además, en las proyecciones "programáticas" las premisas sobre cada componente incorporan las metas establecidas en el PNP de un sexenio determinado, sin que ello implique que el gobierno mexicano adopte como meta de mediano o largo plazos la trayectoria predefinida para los años subsecuentes. Entonces, es posible concluir que el supuesto sobre la migración constante y, por ende de crecimiento económico de $5 \%$, obedeció a una coyuntura políticoeconómica ubicada al inicio del sexenio de Vicente Fox.

\section{Las expectativas y las realidades de la relación economía-migración}

El inicio de la administración de Vicente Fox estuvo lleno de expectativas. En principio, se rompió con 70 años del gobierno de un mismo partido político en el país. Ese sexenio estuvo marcado por una gran esperanza en "el cambio" prometido. Después de los resultados económicos del año 2000 parecía promisorio el futuro de dicha administración y el de México. La economía mexicana se expandió 7\%, rebasando la meta oficial $(4.5 \%)$ y las expectativas de principio de año $(3.5 \%)$. Las exportaciones elevaron su dinamismo, impulsadas por la economía de Estados Unidos y las ventas de petróleo; las importaciones crecieron en igual magnitud, lo que se asoció al vigor de la demanda interna, a pesar de que la política monetaria fue crecientemente restrictiva y de la escasez de crédito bancario. Debido a la recuperación 
del salario real y el aumento del empleo, el consumo se expandió 8\% y la inversión fija 11\% (CEPAL, 2000: 61).

Respecto a la migración, Vicente Fox se pronunció por una política de "fronteras abiertas", la cual fue concebida como una política acorde con la integración regional. Poco tiempo después, a principios de 2001, Vicente Fox y George W. Bush convinieron en que buscarían nuevas respuestas a las realidades de la migración entre ambos países; de manera explícita acordaron que alcanzarían acuerdos de corto y largo plazos que permitieran atender de manera constructiva la migración, y se comprometieron a establecer un esquema ordenado de flujos migratorios (Alba, 2004: 236). Las propuestas específicas parecían promisorias, y de haberse concluido hubiesen generado gran certidumbre en cuanto a la migración entre México y Estados Unidos. Sin embargo los acontecimientos del 11 de septiembre de 2001 cambiaron la historia, y los temas de seguridad se apoderaron del fenómeno migratorio. Asimismo, en ese año la economía mexicana se estancó, en fuerte contraste con la meta original de $4.5 \%$ y con el desempeño del año anterior (7\%). Después de un crecimiento de $1 \%$ en el primer semestre, por primera vez desde 1995 el producto interno bruto comenzó a retroceder $(-1.6 \%)$ durante el tercer trimestre de 2001, determinando una leve contracción para el conjunto del año (CEPAL, 2001: 84).

En virtud de las expectativas antes mencionadas del gobierno mexicano y de lo imprevisible que era un ataque terrorista a Estados Unidos, es posible que al realizar el estudio que sirve de base a los supuestos sobre la migración en las proyecciones poblacionales, los autores esperarían que al menos se tuviera el mismo crecimiento económico que se observó durante la segunda mitad de la década los noventa (PND 2001-2006, 2001: 88). Este periodo corresponde a la administración de Ernesto Zedillo, la cual comenzó con una gran crisis financiera y con el estallido de un movimiento armado (además de la gran cantidad de asesinatos políticos). Es decir, es muy factible que se esperara un crecimiento económico sostenible en una administración que comenzó con mucho optimismo, si se le compara con un periodo en que hubo gran incertidumbre económica y se logró un crecimiento de 5 por ciento.

Sin embargo no ocurrió así. En el sexenio de Fox el crecimiento de la economía fue en descenso y alcanzó un promedio anual de aproximadamente $2.28 \%$. Además no hay evidencia de que México haya tendido a una convergencia económica con Estados Unidos en 
los últimos años. De acuerdo con Alba, la evolución de los productos per cápita de estos países no indica que a raíz del TLC se haya generado alguna tendencia sostenida hacia la convergencia. En efecto, este autor reporta que, de acuerdo con las cifras publicadas por el Banco Mundial, en 1993 el PIB per cápita mexicano equivalía a 16\% del estadounidense y, pasando por varias fluctuaciones, en 2000 el producto mexicano fue 17\% del estadounidense (Alba, 2004: 221).

En suma, dada la evidencia encontrada, no existe ningún sustento empírico que indique que México se encontraba en un camino hacia el crecimiento económico o que en el corto plazo, a partir del 2000, se hubiese podido observar un crecimiento sostenido de 5\%. Tampoco se encontró alguna evidencia que indicara que las economías mexicana y estadounidense estuvieran en un proceso de convergencia como para establecer que en el futuro esto se acentuaría. Por ello, y con la perspectiva del tiempo, se concluye que no existe ningún fundamento empírico que haya llevado al establecimiento del supuesto de tasas de emigración constantes a lo largo del horizonte proyectivo tanto en el ejercicio 2001-2050 como en el ejercicio 2006-2050.

\section{Las variantes de proyección}

En la metodología utilizada para proyectar la población en México se acostumbra elaborar variantes de proyección o escenarios alternativos con el fin de evaluar la incertidumbre asociada a cada componente demográfica y para identificar la repercusión de los supuestos adoptados en el comportamiento futuro de la población. Más allá de las inconsistencias estadísticas de elaborar dichas variantes, ${ }^{5}$ este apartado se enfocará en el análisis de los supuestos sobre la componente migratoria.

Para las proyecciones del periodo 2005-2050 se supusieron dos escenarios alternativos: en el primero las tasas de emigración de los mexicanos se mantendrían constantes hasta 2010 y después descenderían linealmente hasta llegar en 2050 a la mitad de los niveles obser-

${ }^{5} \mathrm{El}$ método de las componentes demográficas, en su versión determinista, contiene dos importantes inconsistencias estadísticas en cuanto al cálculo de variantes de proyección: 1) supone una correlación perfecta entre las componentes y 2) supone una perfecta correlación serial para cada una. Este tipo de supuestos no son reales y causan diversas inconsistencias estadísticas: dos variantes extremas para una variable no son necesariamente extremas para otra variable (Keilman et al., 2002: 410). 
vados en el quinquenio 2000-2005 y, en el segundo, se supuso que las tasas de emigración de mexicanos se mantendrían constantes hasta 2010 y después aumentarían hasta que fueran $50 \%$ superiores. Además se incorporó el supuesto de que las tasas de migración de retorno de mexicanos y de migración neta hacia el resto del mundo se mantendrían invariables a lo largo de la proyección. Para las proyecciones del periodo 2000-2050 sólo se supuso un escenario alternativo: tasas de emigración de mexicanos constantes hasta 2010 y después en descenso lineal hasta llegar en 2050 a la mitad de los niveles observados en el periodo 1995-2000. También se supuso que las tasas de migración de retorno y de migración neta de la población extranjera se mantendrían invariables a lo largo de la proyección.

En este contexto se observa (gráficas 2 y 3 ) que suponer primero tasas constantes y luego descendentes se enmarca en uno de los escenarios de crecimiento más favorable ("C"). Es decir, tanto en las proyecciones 2000-2050 como en las del periodo 2005-2050 se considera como escenario extremo o cota inferior una economía alta y un flujo de remesas creciente a lo largo del horizonte de proyección. Cabe mencionar que en las proyecciones del periodo 2000-2050 sólo se utiliza esta variante, y queda por entendido que no consideraron que la migración pudiera crecer de manera más acelerada; dicho de otra manera, no se reparó en la posibilidad de que el crecimiento económico fuese bajo y el flujo de remesas fuese constante o creciente (como en realidad ocurrió). En cambio, para las proyecciones del periodo 2005-2050 se consideró este escenario como cota superior de la proyección media. Al suponer una tasa de crecimiento constante hasta el año 2010 se considera implícitamente que la tasa de crecimiento económico estará cercana a la meta de crecimiento y que después descenderá por alguna razón. De acuerdo con la gráfica 2 las dos curvas con valores más altos en ningún momento se mantienen constantes durante los primeros 10 años del siglo XXI; al contrario, se observa que a partir del año 1995 su ritmo de cambio es positivo, casi de manera constante, con la forma de una línea recta con pendiente positiva. Así, este último escenario no parece tener sustento empírico alguno, y además se ve un tanto forzado para que, al menos en el corto plazo, proyecte niveles aceptables de crecimiento de la migración. De esta forma, el cálculo de variantes de proyección para la componente migratoria rompe con la motivación inicial referente a no establecer supuestos arbitrarios (Conapo, 2006: 25). 
De acuerdo con José Luis Calva,

el documento "Visión 2030. El México que queremos", dado a conocer [...] por el presidente Felipe Calderón, postula como meta para 2030 que nuestro país alcance un PIB per cápita de 29 mil dólares. Si este objetivo se expresa en dólares corrientes -lo que implicaría que en 2030 el PIB per cápita fuera de 14 mil 166 dólares a valor presente (suponiendo una inflación de 3\% anual en el país emisor del dólar)-, la meta sería un crecimiento del PIB nacional a una tasa media de $3.02 \%$ anual (suponiendo una paridad real peso-dólar constante). Se trataría de una triste resignación a la mediocridad. Por el contrario, si la meta de 29 mil dólares de PIB per cápita para 2030 se expresa a valor presente, la economía mexicana tendría que crecer a una tasa media de $6.1 \%$ anual. Se trataría de un objetivo halagüeño, pero razonablemente factible, próximo al potencial de crecimiento que realmente tiene la economía mexicana [...] En un escenario no deseable (denominado "inercial"), el documento "Visión 2030" proyecta un PIB per cápita de 11 mil 485 dólares para 2030, lo que implicaría un crecimiento del PIB nacional a una tasa media de $2.12 \%$ anual. No es algo improbable: es casi igual a la tasa media de crecimiento observada en el gobierno del presidente Fox (2.28\% anual durante el sexenio 20012006). Si esta última tasa se mantuviera en el futuro, México apenas alcanzaría un PIB per cápita de 11 mil 923 dólares en 2030 [Calva, 2007].

En virtud de lo anterior, se concluye que, al igual que los supuestos en torno a la proyección media, las valoraciones de la incertidumbre asociada a la migración futura se encuentran relacionadas con la esperanza de alcanzar los objetivos económicos, determinados en la coyuntura política actual, en el corto plazo.

\section{Reflexiones finales}

En este trabajo se han estudiado los supuestos subyacentes a la proyección de la componente migratoria. Para ello se analizaron las fuentes citadas en la metodología prospectiva del Conapo para los periodos 2001-2050 y 2006-2050, y se les vinculó con la literatura relacionada. Se encontró que en los supuestos sobre la proyección media de la migración internacional subyace la teoría económica neoclásica. En los ejercicios prospectivos analizados en este trabajo se supone que en el futuro, dado un crecimiento económico sostenido de $5 \%$, la migración crecerá a una tasa constante cercana a 0.22 , por lo menos durante los primeros diez años del siglo XXI. Cabe mencio- 
nar que este supuesto se encuentra vinculado con las expectativas de las coyunturas político-económicas de los dos primeros gobiernos mexicanos del siglo XXI y con el crecimiento económico alcanzado en el último quinquenio del siglo pasado.

Así, se concluye que para determinar la tasa de crecimiento futura de la migración se consideraron las dimensiones económicas, sociales y demográficas; esta última de manera implícita, ya que la estructura por edad y sexo forzosamente deben ser incluidas en las proyecciones de cada componente. Además, no se encontró evidencia de que haya sido considerada la dimensión de políticas específicas de la migración para proyectar la migración futura de México. Al establecer los supuestos sobre la tasa de migración futura, primordialmente en el marco de la teoría económica neoclásica, el papel de las políticas ante el fenómeno migratorio juega en el sentido de afectarlo de manera colateral enfocando sus esfuerzos en lo que se considera que son los determinantes económicos del mismo. Por ello, ante la ausencia de políticas explícitas sobre la migración en los últimos años, no se consideró que pudiesen existir en el futuro. Es decir, se puede asegurar que existe un factor de política dentro de las proyecciones de la migración pero no se encuentra directamente relacionado con ésta, sino con las metas económicas que tiene el Estado y la forma en que pueden afectar las tasas de emigración.

Si se enmarca lo anterior dentro de las proyecciones "programáticas”, es razonable el marco teórico en el que se suscriben los supuestos, más no los supuestos en sí. Es claro que para la planeación de programas, la asignación de presupuestos y la evaluación de sus logros, los tomadores de decisiones deben conocer qué metas deben alcanzar en el futuro, pero también es necesario valorar la posibilidad de alcanzar dichas metas en el corto, mediano y largo plazos. En la medida en que se consideren los posibles escenarios futuros se podrán tomar decisiones más acertadas. Por ello, las proyecciones "programáticas", como instrumento de política, son una condición necesaria más no suficiente para la planeación de un país. Además, de acuerdo con la definición de proyección programática propuesta por Partida y Tuirán (2002), debe ampliarse en el sentido de que no sólo se incluyan metas sobre la fecundidad sino, como se ha demostrado en este artículo, también sobre la migración.

Ahora bien, respecto al cálculo de las variantes de proyección se encontró que, debido a que no existe ningún sustento empírico que indique que el crecimiento de la economía pueda ser mejor que el del sexenio anterior, al igual que los supuestos en torno a la proyección 
media, las valoraciones de la incertidumbre asociada a la migración futura están relacionadas con la esperanza de alcanzar los objetivos económicos en el corto plazo, determinados en la coyuntura política del inicio del gobierno de Felipe Calderón. El sexenio de Vicente Fox inició con mucho optimismo acerca del futuro del país y sesgó las previsiones nacionales, en particular sobre el futuro demográfico. $\mathrm{Al}$ respecto la historia ha dejado una amarga lección. El gobierno de Calderón se inició con una sociedad polarizada y con un cuestionamiento sobre sus instituciones nacionales, además de un panorama económico global un tanto incierto. Por ello no es factible incluir sólo metas políticas en la prefiguración del futuro de una nación ya que es muy difícil que se alcancen. Éste tan sólo debe ser un escenario a considerar en un análisis más integral sobre el futuro demográfico de México.

La realidad ha superado las expectativas de las proyecciones, pero no por los motivos que se deseaban, sino por el efecto inesperado en la disminución del saldo neto migratorio en términos absolutos atribuido a la recesión económica mundial que inició en 2006. De acuerdo con Passel (2011: 17-19) la tendencia del fenómeno migratorio de México cambió después de 2007 al iniciar la recisión económica, ya que

los mexicanos se vieron afectados por la contracción de la economía estadounidense. Con el colapso del auge inmobiliario, iniciado en 2006, el sector de la construcción, que tradicionalmente emplea a un gran número de trabajadores mexicanos, cayó antes que el resto de la economía. En 2007, el flujo de inmigrantes mexicanos fue sólo la mitad de lo que había sido dos años antes (280 mil). Los números siguieron cayendo hasta llegar a cerca de 155 mil en 2009 [...] [la] inmigración bruta permaneció virtualmente sin cambios en 2010 y registró su nivel más bajo de las últimas dos décadas [...] durante los últimos tres años, las tasas de desempleo en Estados Unidos alcanzaron el nivel más alto observado desde hace mucho tiempo [...] [por] primera vez desde los ochenta, el número de mexicanos indocumentados decreció.

\section{El autor atribuye este estancamiento}

principalmente a una gran caída en el número de nuevos inmigrantes y no al incremento de las salidas del país. De hecho, la probabilidad de retorno a México para los migrantes indocumentados está en sus niveles más bajos [...] [la] inmigración neta de residentes mexicanos a Estados Unidos cayó a casi cero como resultado del equilibrio en el número de entradas y salidas. 
En este artículo se demuestra lo sensibles que son las proyecciones de población cuando no se considera a los fenómenos demográficos como intrínsecamente inciertos, a pesar de que cambian más lentamente que otros. La aproximación para establecer los supuestos sobre la migración futura de México propuesta por Tuirán y sus colaboradores (2000a y 2000b) es realmente muy novedosa y, como tal, debe ser el punto de partida para otras propuestas más elaboradas y robustas. Si se incluyera el factor incertidumbre en un ejercicio similar de manera que pudiese medir la incertidumbre asociada, es muy probable que se obtengan resultados más robustos y objetivos.

Es muy fácil caer en supuestos arbitrarios en los modelos deterministas y más cuando se utilizan para proyectar los fenómenos demográficos. Sin embargo, en la actualidad se cuenta tanto con herramientas teórico-metodológicas como con herramientas computacionales que sirven para determinar el nivel de incertidumbre del fenómeno en cuestión. Para el caso de la migración internacional se han desarrollado menos técnicas estocásticas que para la mortalidad y la fecundidad. El posible retraso metodológico se debe a la falta de información confiable, pero en los trabajos de Hyndman y Booth (2008), Bijak (2011) y García Guerrero (2014) se presentan alternativas viables, aunque el marco del que parten es un tanto distinto del modelo TPA. En efecto, analíticamente los primeros tres trabajos modelan y proyectan la migración como una variable que sólo depende del tiempo, y toda la información sobre ella está contenida en ella misma. Por otro lado el modelo TPA modela la migración como una variable que depende de ciertas variables económicas de acuerdo con un marco teórico determinado.

Por ejemplo, en las gráficas 4 y 5 se muestra un ejercicio de proyección estocástica de la migración masculina y femenina, respectivamente, a partir de las mismas estimaciones calculadas por la Sociedad Mexicana de Demografía (2011). El método utilizado es una variación del modelo de Lee-Carter que se explica en García Guerrero (2014). Las franjas representan el intervalo de $95 \%$ de probabilidad de la proyección 1991-2010 realizada a partir de la información estimada entre 1960 y 1990, y la línea negra es la estimación entre los años 1990 y 2010. En ambas figuras se muestra que si bien con un método estocástico no se pronostica puntualmente la migración neta, sí se refleja adecuadamente la incertidumbre asociada a esta variable demográfica, además de que se libran los problemas de inconsistencia estadística mencionados anteriormente. Otra ventaja de este método es que pro- 


\section{GRÁFICA 4}

Comparativo entre el pronóstico estocástico de la migración neta mexicana y estimaciones; hombres, 1990-2010

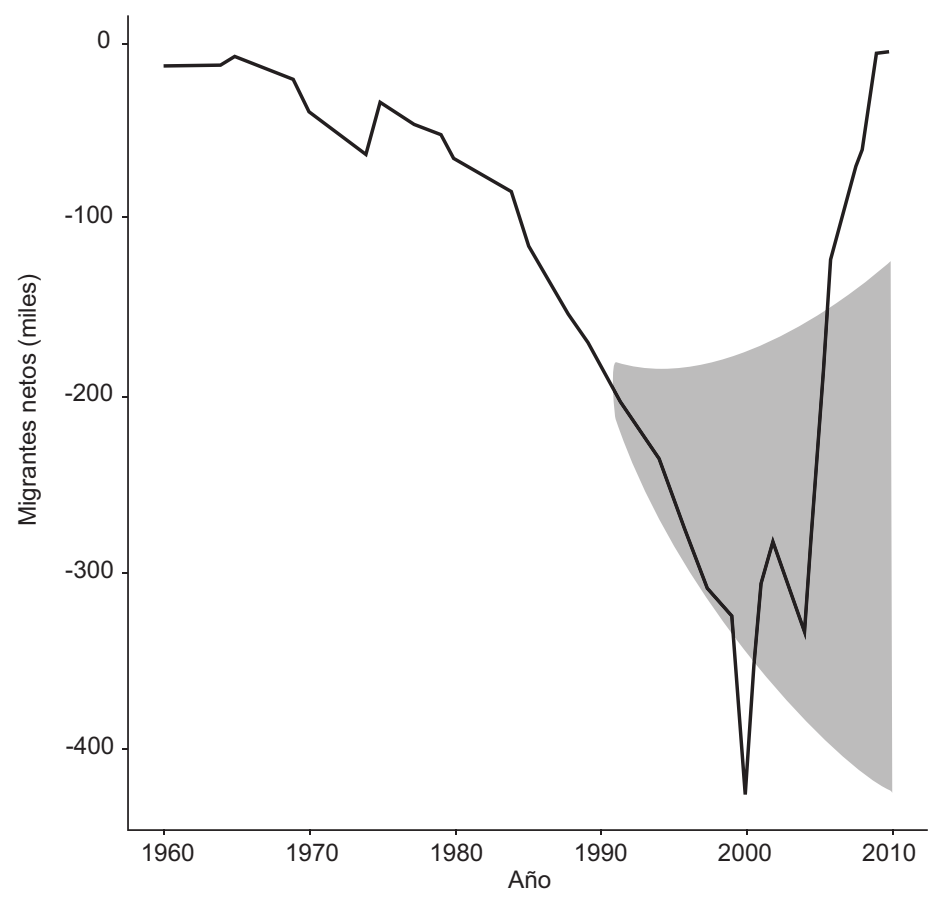
2011.

FUENTE: Cálculos propios con base en la conciliación demográfica de Somede,

nostica la migración neta total a partir de la migración neta estimada por edades simples, lo cual evita emplear supuestos adicionales acerca de la estructura por edades.

El modelo TPA puede mejorarse si se emplean modelos de vectores autorregresivos (VAR) o algún otro en el que las variables independientes sean pronosticadas con alguna técnica de series de tiempo y luego incorporadas en un modelo de simulación con el que puedan calcularse los intervalos de confianza correspondientes. Otra forma de mejorar el modelo es utilizando técnicas bayesianas en donde se use el conocimiento a priori que se tiene acerca de los parámetros del modelo de regresión. Indudablemente el modelo TPA puede enrique- 


\section{GRÁFICA 5}

Comparativo entre el pronóstico estocástico de la migración neta mexicana y estimaciones; mujeres, 1990-2010

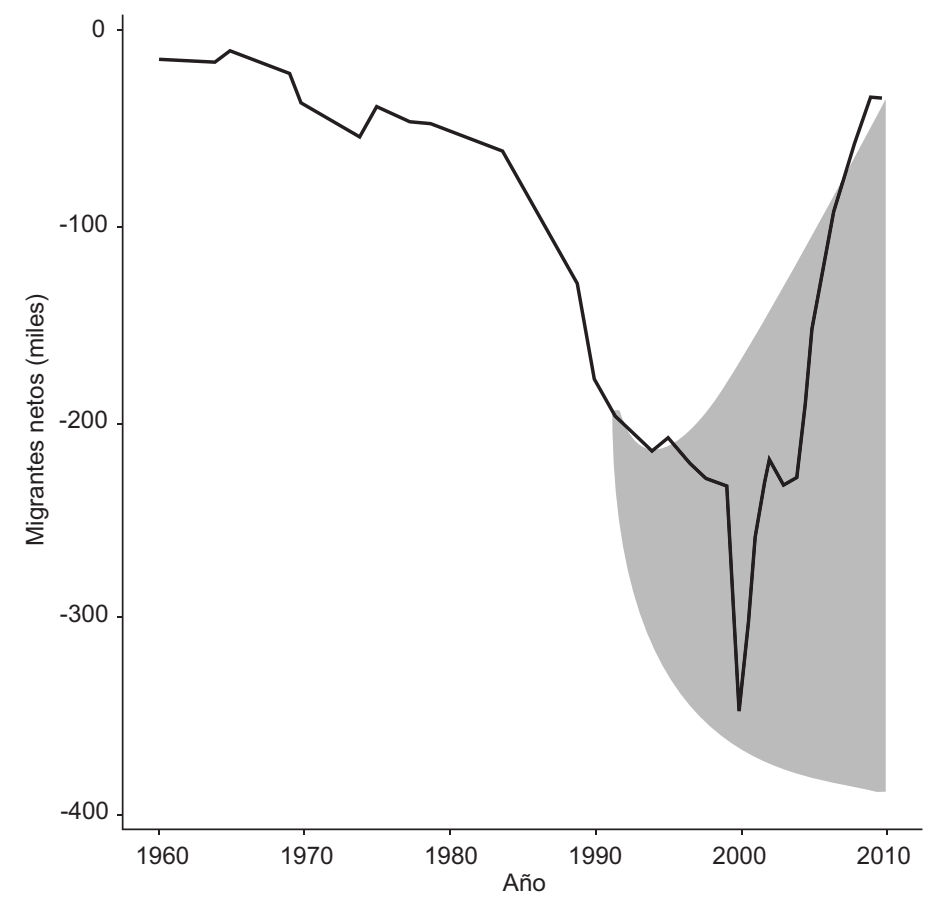
2011.

FuENTE: Cálculos propios con base en la conciliación demográfica de Somede,

cerse de muchas maneras, pero para capitalizar eficientemente dicho conocimiento es necesario permitir y garantizar que los resultados obtenidos sean replicables.

\section{Bibliografía}

Alba, Francisco (2004), "El Tratado de Libre Comercio, la migración y las políticas migratorias”, en E. Casares y H. Sobarazo (comps.), Diez años del TLCAN en México. Una perspectiva analítica, México, Fondo de Cultura Económica, pp. 215-242. 
Alba, Francisco (2006), "Cambios y continuidades de las respuestas gubernamentales frente a la emigración mexicana”, en José Luis Lezama y José B. Morelos (coords.), Población, ciudad y medio ambiente en el México contemporáneo, México, Centro de Estudios Demográficos, Urbanos y Ambientales, El Colegio de México, pp. 277-306.

Bijak, Jakub (2011), Forecasting International Migration in Europe: A Bayesian View, Dordrecht, Springer (The Springer Series on Demographic Methods and Population Analysis, 24).

Calva, José Luis (2007), "Metas de crecimiento 2030”, artículo periodístico, El Universal, 25 de mayo. Disponible en $<$ http://www.eluniversal.com.mx/editoriales/37683.html >.

CEPAL (2000), Balance preliminar de las economías de América Latina y el Caribe 2000, Santiago de Chile, Comisión Económica para América Latina y el Caribe, Naciones Unidas.

CEPAL (2001), Balance preliminar de las economías de América Latina y el Caribe 2001, Santiago de Chile, Comisión Económica para América Latina y el Caribe, Naciones Unidas.

CMMI (2005), Las migraciones en un mundo interdependiente: nuevas orientaciones para actuar. Informe de la Comisión Mundial sobre las Migraciones Internacionales, Suiza, Comisión Mundial sobre las Migraciones Internacionales.

Conapo (2003), "Proyecciones de la población de México, de las entidades federativas, de los municipios y de las localidades, 2000-2050", documento metodológico, México, Consejo Nacional de Población.

Conapo (2006), Comunicado de prensa 37/06, Consejo Nacional de Población, 7 de septiembre $<$ http://www.conapo.gob.mx $>$.

Conapo (2008), "Proyecciones de la población en México, de las entidades federativas, de los municipios y de las localidades 2005-2050", documento metodológico, México, Consejo Nacional de Población.

Durand, Jorge (2006), "Programas de Trabajadores Temporales", México, Consejo Nacional de Población (Temas de Migración).

Escobar Latapí, Agustín et al. (1999), "Factores que influyen en la migración", en Estudio binacional México-Estados Unidos sobre migración, México, Secretaría de Relaciones Exteriores.

García Guerrero, Víctor Manuel (2014), Proyecciones y políticas de población en México, México, Centro de Estudios Demográficos, Urbanos y Ambientales, El Colegio de México.

Hyndman, Rob y H. Booth (2008), "Stochastic Population Forecasts Using Functional Data Models for Mortality, Fertility and Migration", International Journal of Forecasting, vol. 24, núm. 3, pp. 323-342.

Keilman, Nico, D. Quang y A. Hetland (2002), "Why Population Forecast Should Be Probabilistic. Illustrated by the Case of Norway", Demographic Research, vol. 6, núm. 15, pp. 409-452.

Kritz, Mary y H. Zlotnik (1992) "Global Interactions: Migration Systems, Processes, and Policies”, en M. Kritz, L. Lim y H. Zlotnik (coords.), In- 
ternational Migration Systems. A Global Approach, Oxford, Clarendon Press, pp. 1-16.

Lee, Ronald (1998), "Probabilistic Approaches to Population Forecasting", en Wolfgang Lutz, James W. Vaupel y Dennis A. Ahlburg (coords.), Frontiers of Population Forecasting, suplemento de Population and Development Review, vol. 24, pp. 156-190.

Massey, Douglas et al. (2000), "Teorías sobre la migración internacional: una reseña y una evaluación”, Trabajo, vol. 2, núm. 3, pp. 5-49.

Passel, Jeffrey S. (2011), "Flujos migratorios México-Estados Unidos de 19902010: un análisis preliminar basado en las fuentes de información estadounidenses”, Coyuntura Demográfica. Revista sobre los Procesos Demográficos en México Hoy, núm. 1, México, Somede.

Partida, Virgilio y Rodolfo Tuirán (2002), "Evolución futura de la población mexicana: envejecimiento y bono demográfico”, en Brígida García (coord.), Población y sociedad al inicio del siglo XXI, México, El Colegio de México, pp. 25-54

PND 2001-2006 (2001), "Plan Nacional de Desarrollo 2001-2006”, Diario Oficial de la Federación, México, 30 de mayo.

Rogers, Andrei (1995), Multiregional Demography. Principles, Methods and Extensions, Nueva York, John Wiley and Sons.

SPP, Conapo y Celade (1983), México: estimaciones y proyecciones de la población, 1950-2000, México, Secretaría de Programación y Presupuesto / Consejo Nacional de Población / Centro Latinoamericano de Demografía.

Smith, Stanley, J. Tayman y D. Swanson (2001), State and Local Population Projections. Methodology and Analysis, Nueva York, Kluwer Academic-Plenum Publishers (The Plenum Series on Demographic Methods and Population Analysis).

Somede (2011), Conciliación demográfica de México y entidades federativas, 19902010, México, Sociedad Mexicana de Demografía.

Tuirán, Rodolfo, Virgilio Partida y José Luis Ávila (2000a), "Las causas de la migración hacia Estados Unidos”, en Rodolfo Tuirán (coord.), Migración México-Estados Unidos. Presente y futuro, México, Conapo, pp. 30-34.

Tuirán, Rodolfo, Virgilio Partida y José Luis Ávila (2000b), "Crecimiento económico, libre comercio y migración”, en Rodolfo Tuirán (coord.), Migración México-Estados Unidos. Presente y futuro, México, Conapo, pp. 54-76.

Tuirán, Rodolfo, Jorge Santibáñez y Rodolfo Corona (2006), "El monto de las remesas familiares en México: ¿mito o realidad?”, Papeles de Población, núm. 50, pp. 147-169.

U.S. Commission on the Immigration Reform (1997), A report of the Binational Study on Migration, U.S. Commision on the Immigration Reform. Disponible en <http://www.utexas.edu/lbj/uscir/binational.html $>$. 


\section{Acerca del autor}

Víctor Manuel García Guerrero es doctor en Estudios de Población por El Colegio de México; parte de su investigación doctoral la realizó en el World Population Program del International Institute for Applied Systems Analysis en Austria y tomó cursos en el Max Planck Institute for Demographic Research en Alemania. Estudió Actuaría, la maestría en Investigación de Operaciones y un diplomado en Econometría Avanzada en la UNAM. Fue profesor investigador de la Flacso sede México y profesor de tiempo parcial en el ITAM y en la Facultad de Ciencias de la UNAM. Ha sido asesor y consultor en métodos demográficos para el Conapo y para distintos despachos de consultoría y bancos. Sus temas de investigación son: demografía formal y sus aplicaciones, estimaciones y pronósticos demográficos y sus relaciones con las políticas de población. Entre sus publicaciones más recientes destaca el libro Proyecciones y políticas de población en México (El Colegio de México, 2014). Es candidato a investigador nacional por el Sistema Nacional de Investigadores del Conacyt. Actualmente es profesor investigador y coordinador de la maestría en Demografía del Centro de Estudios Demográficos, Urbanos y Ambientales de El Colegio de México. 